\title{
A Comparative Evaluation of Antigen-Specific Sandwich Immunoassay and Indirect Immunofluorescence Assay (IIF) in Detecting Antineutrophil Cytoplasmic Antibodies: Are We Ready to Replace IIF with ELISA as the Primary Screening Method?
}

\author{
Sangeeta Deka ${ }^{1}$ Deepjyoti Kalita ${ }^{1, \odot}$ Ravi Shankar $^{1}$ \\ ${ }^{1}$ Department of Microbiology, All India Institute of Medical \\ Sciences, Rishikesh, Uttarakhand, India \\ 2Department of Pulmonary Medicine, All India Institute of Medical \\ Sciences, Rishikesh, Uttarakhand, India
}

\section{U. Sasi Rekha ${ }^{1}$ Girish Sindhwani ${ }^{2}$}

\begin{abstract}
Address for correspondence Deepjyoti Kalita, MD, PhD, Department of Microbiology, All India Institute of Medical Sciences Rishikesh, Virbhadra Road, Rishikesh 249203, Uttarakhand, India (e-mail: dkalita@gmail.com,deep.micro@aiimsrishikesh.edu.in).
\end{abstract}

J Lab Physicians 2021;13:286-290.

\begin{abstract}
Keywords

- ANCA

- screening

- PR3-ANCA

- MPO-ANCA

- ELISA

- indirect

immunofluorescence
\end{abstract}

Background Antineutrophil cytoplasmic antibodies (ANCA) are important biomarkers in the diagnosis of ANCA-associated vasculitis, and indirect immunofluorescence (IIF) had been the method of choice for its detection from the very beginning. However, international consensus on ANCA testing (2017) advocates the use of high-quality immunoassays as the primary screening method. The purpose of this study was to evaluate the diagnostic performance of enzyme-linked immunosorbent assay (ELISA) compared to IIF in detecting ANCA.

Methods One-hundred eighty-nine serum samples of suspected or known cases of systemic vasculitis were screened for ANCA by IIF and proteinase-3- and myeloperoxidase-ELISA. In IIF, positive results were further divided into cytoplasmic pattern of ANCA and perinuclear pattern of ANCA, depending upon the pattern of fluorescence. McNemar's chi-squared test was applied to check the equality of proportions of positive results, and Kappa statistics was used to measure the agreement between the two methods. Diagnostic performance of ELISA was evaluated taking IIF as reference.

Results IIF detected ANCA in $17.5 \%$ cases and ELISA detected it in $11.6 \%$ cases. A good agreement between the overall performance of ELISA and IIF was observed ( $K$-value: 6.8, p-value: <001). However, a significant difference in the proportion of positive results by the two methods was observed in McNemar's test (two-sided $p$-value: 0.007). Taking IIF as standard, ELISA showed $60.6 \%$ sensitivity, $98.7 \%$ specificity, and predictive value of positive and negative results of 90.9 and $92.2 \%$, respectively.

Conclusion The new generation antigen-specific ELISAs had high specificity but the chances of missing cases in primary screening due to the low sensitivity and high false negativity $(39.4 \%)$ need to be dealt with. published online July 10,2021
DOI https://doi.org/

$10.1055 / \mathrm{s}-0041-1731945$ ISSN 0974-2727 (c) 2021. The Indian Association of Laboratory Physicians.

This is an open access article published by Thieme under the terms of the Creative Commons Attribution-NonDerivative-NonCommercial-License, permitting copying and reproduction so long as the original work is given appropriate credit. Contents may not be used for commercial purposes, or adapted, remixed, transformed or built upon. (https://creativecommons.org/licenses/by-nc-nd/4.0/).

Thieme Medical and Scientific Publishers Pvt. Ltd. A-12, 2nd Floor, Sector 2, Noida-201301 UP, India 


\section{Introduction}

Screening for antineutrophil cytoplasmic antibody (ANCA) is instrumental in the diagnosis of systemic necrotizing small-vessel vasculitis namely, granulomatosis with polyangiitis (previously called Wegener's granulomatosis), microscopic polyangiitis, and eosinophilic granulomatosis with polyangiitis (previously called Churg-Strauss syndrome), which are collectively termed as ANCA-associated vasculitis (AAV). ${ }^{1}$ However, over time the clinical spectrum of AAV has widened. ${ }^{2}$ On indirect immunofluorescence (IIF), antibodies that show cytoplasmic fluorescent pattern and target mostly proteinase-3 (PR3) antigen are called C-ANCA, while the others showing a perinuclear pattern that targets mainly myeloperoxidase (MPO) antigen are called P-ANCA. ${ }^{3}$ Serologic classification of AAV into PR3-ANCA and MPO-ANCA correlates with several disease characteristics.

Ever since Davies et $\mathrm{al}^{4}$ first reported the presence of antibodies producing diffuse cytoplasmic staining of neutrophils by IIF techniques in patients with segmental necrotizing glomerulonephritis, the laboratory diagnosis of ANCA has evolved manifold. ${ }^{5}$ The first-generation commercial enzyme-linked immunosorbent assay (ELISA) marketed in 1991 detected ANCA by direct antigen binding. Subsequently, it was improved to capture-based antigen binding (second-generation) and anchor-based antigen binding (third-generation) ELISA and other assays. ${ }^{5,6}$ Similarly, IIF has also evolved from using a plain neutrophil substrate to the antigen-specific biochip and microbead technology. Parallel to ELISA and IIF, novel, sensitive, and automated technologies, such as fluoroenzyme immunoassays, chemiluminescence assays, solid-phase assays (dot and line immunoassays), and laser-bead immunoassays have also been introduced. ${ }^{5-7}$ Nonetheless, controversies remain regarding the methodologic accuracy of different assays. ${ }^{8}$ Despite the disagreements, ANCA testing is widely used for the diagnosis of AAV. Since its inception, IIF had been regarded as the gold standard method in ANCA screening. However, a 2016 study by the European Vasculitis Study Group questioned the role of IIF in ANCA screening and claimed a high diagnostic performance of PR3-ANCA and MPO-ANCA by immunoassay. ${ }^{9}$

Henceforth, this study aimed to compare sandwich ELISA's diagnostic performance (using both native and recombinant antigen) with IIF to detect ANCA in systemic small-vessel vasculitis.

\section{Materials and Methods}

This hospital-based prospective study was conducted at All India Institute of Medical Sceinces, Rishikesh, Uttarakhand, India, between August 2018 and January 2019. The study was approved after it was ethically reviewed by the institutional ethics committee of our institute. We prospectively collected 189 blood samples of newly suspected or known systemic necrotizing small vasculitis patients over 5 months (August 1-December 31, 2018) referred to serology laboratory (under Microbiology) for testing ANCA. The referral for ANCA testing came from various specialist physicians, including rheumatologists, nephrologists, neurologists, and general medicine experts. Unlabeled, inadequate, hemolyzed, lipemic, and leaking blood samples were excluded. Sera of patients without specific signs and symptoms of systemic small-vessel vasculitis were also excluded from the study.

Blood was collected by venipuncture in sterile plain vials. Serum was separated by centrifugation, and aliquoted serum was stored at $-20^{\circ} \mathrm{C}$ until further processed. The serum samples finally included in the study were subjected to IIF (EUROIMMUN, Lübeck, Germany) and then monospecific sandwich third-generation ELISAs namely-anti-PR3-hn-hr ELISA-immunoglobulin G (IgG) and anti-MPO-ELISA-IgG (both EUROIMMUN Medizinsche Labordiagnostika AG, Lübeck, Germany) to detect the presence of ANCA.

The ELISA testing kits contained microtiter strips, each with eight break-off reagent wells coated with a mixture of purified recombinant PR3 and native PR3 (in anti-PR3-hn-hr ELISA, IgG) and purified-MPO (in anti-MPO-ELISA, IgG) antigens. Controls and prediluted sera were added to the different wells and incubated to allow any anti-PR3 and anti-MPO antibodies (if present) to separately bind to the coated antigens. A second incubation is performed using an enzyme-labeled anti-human IgG (enzyme conjugate) catalyzing a color reaction to detect the bound antibodies. A chromogenic substrate was added to determine the extent of enzyme activity by measuring color intensity by spectrometry. The cutoff used for both tests (PR3 and MPO) was 20 relative units (RU)/mL.

The IIF diagnostic kit slides used ethanol-fixed buffy coat human neutrophils combined with formalin-fixed neutrophils and HEp-2 cells to better discriminate between P-ANCA and ANA. The patient sera were diluted in $1 / 10$ proportion and added to the three wells, and incubated. If the sera contained ANCA, specific antibodies of IgG, IgA, and IgM got attached to the antigens. The bound antibodies were then stained with FITC (green)-labeled antihuman antibodies and observed under the fluorescence microscope. The slides were noted as positive if fluorescence was seen. If granular fluorescence distributed regularly over the entire cytoplasm of the granulocytes leaving the cell nuclei free was observed, it was noted as C-ANCA (cytoplasm pattern). If a smooth fluorescence wrapped around the granulocytes' cell nuclei was observed, it was reported as P-ANCA (perinuclear pattern). P-ANCA was further confirmed by cytoplasmic staining on formalin-fixed neutrophils and divided into formalin-sensitive and formalin resistant P-ANCA.

\section{Statistics}

Collected data were analyzed and interpreted statistically using SPSS v.23.0 (IBM, NY, United States). Descriptive statistics function was used to find out the frequencies in each category. McNemar's chi-squared test was applied for checking equality in the proportions of a positive outcome by the two methods: IIF and ELISA (dependent variable with the dichotomous outcome). ${ }^{10}$ It was also applied to check equality of P-ANCA (IIF) versus anti-MPO-ELISA and C-ANCA(IIF) versus anti-PR3-ELISA. For assessing agreement, we compared the binary outcomes of the two methods using the kappa statistics. In all the statistical tests, a $p$-value of less than 0.05 was considered significant. The indices of sensitivity, specificity, 
and the predictive value of the positive and negative tests were calculated as described. ${ }^{10}$

\section{Results}

Of the 189 samples tested, 33 were detected to have ANCA by IIF (17.5\%) and 22 by ELISA (11.6\%). Twenty cases (10.6\%) came positive in both the methods employed. However, 13 IIF positive samples did not show a positive ELISA result, and two negative cases under IIF were positive in ELISA (one anti-PR3 and one anti-MPO-ELISA; - Table 1).

The 33 IIF positives revealed a C-ANCA pattern in 20 cases and a P-ANCA pattern in 13 cases (seven formalin resistant and six formalin sensitive) (-Fig. 1). However, in the ELISA test $11(5.8 \%)$ patients showed raised antibodies ( $\geq 20 \mathrm{RU} / \mathrm{mL}$ ) against PR3 and 12 (6.3\%) against MPO. One sample was positive for antibodies against both PR3 and MPO (- Table 1).

McNemar's chi-squared test showed a statistically significant change in overall positivity proportions by IIF and ELISA (two-sided p-value: 0.007). However, checking individually between P-ANCA (by IIF) and anti-MPO-ELISA, no significant difference was observed (two-sided $p$-value: 0.100; - Table 2) Kappa statistics showed good agreement between overall IIF and ELISA results ( $K$-value: 0.683, $p$-value: <0.001); and P-ANCA and anti-MPO-ELISA ( $K$-value: $0.700, p$-value: $<0.001)$. But moderate-to-fair agreement was observed between C-ANCA and anti-PR3-ELISA ( $K$-value: 0.477, $p$-value: <0.001; - Table 2). ${ }^{10}$

Table 1 Detection of antineutrophil cytoplasmic antibodies by indirect immunofluorescence and enzyme-linked immunosorbent assay

\begin{tabular}{|c|c|c|c|}
\hline Test & Interpretation & $n$ & $\%$ \\
\hline \multicolumn{4}{|c|}{ Indirect immunofluorescence } \\
\hline & Positive & 33 & 17.5 \\
\hline & Negative & 156 & 82.5 \\
\hline \multicolumn{4}{|c|}{ Pattern of positive IIF } \\
\hline \multirow[t]{2}{*}{ C-ANCA } & Positive & 20 & 10.6 \\
\hline & Negative & 169 & 89.4 \\
\hline \multirow[t]{3}{*}{ P-ANCA } & FS & 6 & 3.2 \\
\hline & FR & 7 & 3.7 \\
\hline & Negative & 176 & 93.1 \\
\hline \multicolumn{4}{|c|}{ Enzyme-linked immunosorbent assay } \\
\hline & Positive & 22 & 11.6 \\
\hline & Negative & 167 & 88.4 \\
\hline \multicolumn{4}{|c|}{ Type of ELISA } \\
\hline \multirow[t]{2}{*}{ Anti-PR3 ELISA } & Positive & 11 & 5.8 \\
\hline & Negative & 178 & 94.2 \\
\hline \multirow[t]{2}{*}{ Anti-MPO ELISA } & Positive & 12 & 6.3 \\
\hline & Negative & 177 & 93.7 \\
\hline
\end{tabular}

Abbreviations: ANCA, antineutrophil cytoplasmic antibodies; C-ANCA, cytoplasmic pattern of ANCA; FR, formalin resistant; FS, formalin sensitive; MPO, myeloperoxidase; P-ANCA, perinuclear pattern of ANCA; PR3, proteinase 3.
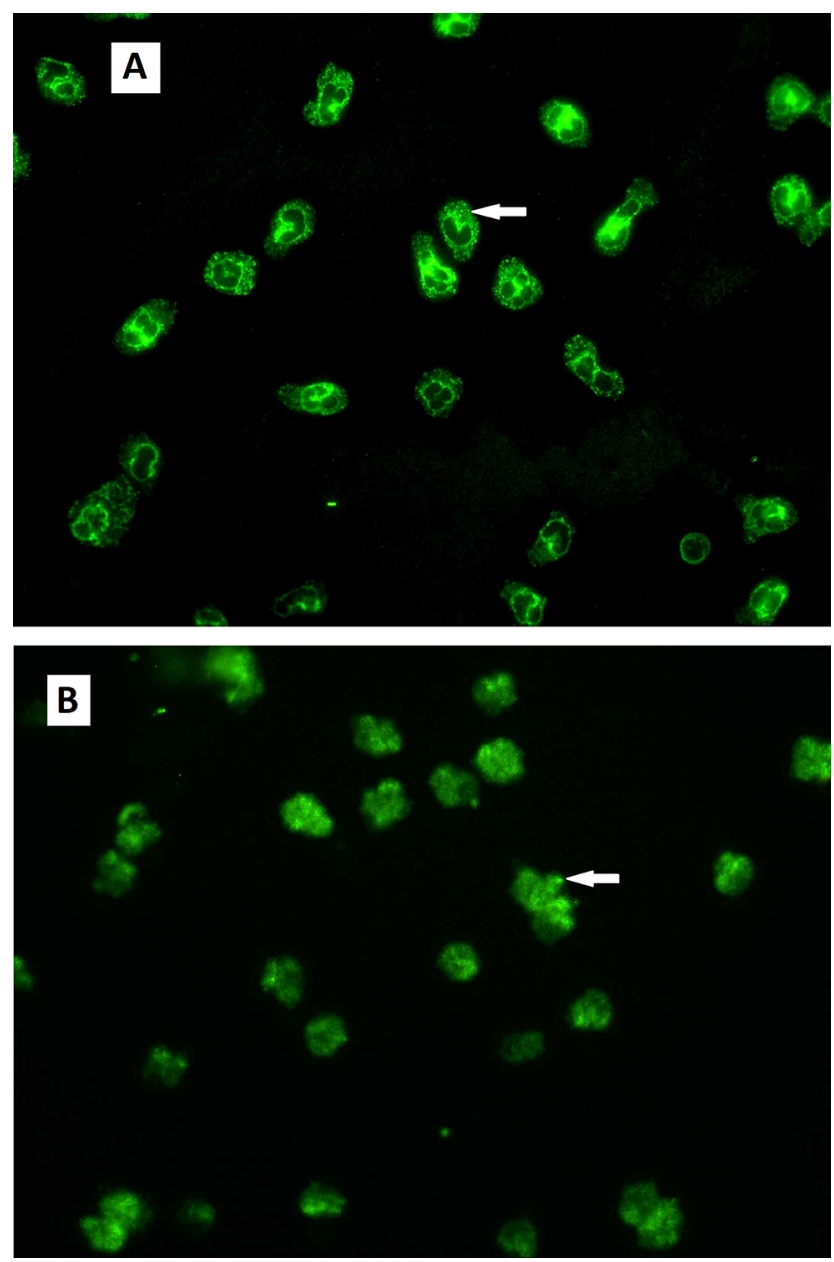

Fig. 1 Appearance of antineutrophil cytoplasmic antibodies in indirect immunofluorescence: (A) cytoplasmic pattern of ANCA (arrow) and (B) perinuclear pattern of ANCA on ethanol-fixed human granulocytes (arrow).

The diagnostic measurements of ELISA in detecting ANCA, with IIF as the reference method, are summarized in - Table 3. The overall ELISA performance had a specificity of $98.7 \%$ and a sensitivity of $60.6 \%$ relative to IIF. Binary results (positive/negative) in the detection of ANCA by IIF and ELISA are tabulated in Supplementary Table S1 (online only).

\section{Discussion}

IIF has been the method of choice for screening ANCA ever since it was first detected. But IIF has its limitations as it requires considerable experience, and it is subjective. According to the International Consensus Statement on Testing and Reporting ANCA (1999), IIF must be performed on all serum samples for screening ANCA in "new" patients, and positive results obtained with IIF should be corroborated with positive antigen-specific ELISA results for PR3 and MPO antigens to support the diagnosis of ANCA-associated small-vessel vasculitis. ${ }^{11}$ But the current revised guidelines (2017) propose that high-quality immunoassays can be used as the primary screening method for patients suspected of having the granulomatosis with polyangiitis and microscopic polyangiitis, 
Table 2 Results of McNemar's chi-squared test and Kappa statistics to check equality and measurement of agreement between the observed outcome of the testing methods under study

\begin{tabular}{|l|l|l|l|l|}
\hline \multirow{2}{*}{} & \multicolumn{2}{|c|}{ McNemar's test } & \multicolumn{2}{c|}{ Kappa agreement } \\
\cline { 2 - 5 } & OR & Exact sig. (2-sided) & K-value & Approx. sig. \\
\hline IIF versus ELISA & 118.4 & 0.007 & 0.683 & 0.000 \\
\hline C-ANCA versus anti-PR3-ELISA & 36.8 & 0.035 & 0.477 & 0.000 \\
\hline P-ANCA versus anti-MPO-ELISA & 129.7 & 1.000 & 0.700 & 0.000 \\
\hline
\end{tabular}

Abbreviations: C-ANCA, cytoplasmic pattern of ANCA; MPO-ELISA, myeloperoxidase-enzyme-linked immunosorbent assay; P-ANCA, perinuclear pattern of ANCA; PR3-ELISA, proteinase 3-enzyme-linked immunosorbent assay; K-value, Kappa coefficient; OR, odds ratio; Sig., p-value.

Table 3 Diagnostic measurements of enzyme-linked immunosorbent assay for detection of antineutrophil cytoplasmic antibodies, according to indirect immunofluorescence

\begin{tabular}{|l|l|l|l|l|l|l|l|}
\hline & \% pos & FP & FN & SEN & SPF & PPV & NPV \\
\hline $\begin{array}{l}\text { Overall ELISA } \\
\text { outcome }\end{array}$ & 11.6 & 1.3 & 39.4 & 60.6 & 98.7 & 90.9 & 92.2 \\
\hline Anti-PR3-ELISA & {$[7.4-9.1]$} & {$[0.2-4.5]$} & {$[22.9-57.8]$} & {$[42.1-77.1]$} & {$[95.4-99.8]$} & {$[70.8-98.9]$} & {$[87.1-95.8]$} \\
& {$[2.5-9.2]$} & {$[0.4-5.1]$} & {$[36.1-80.9]$} & {$[19.1-63.9]$} & {$[94.9-99.6]$} & {$[39.0-93.9]$} & {$[88.5-96.5]$} \\
\hline $\begin{array}{l}\text { Anti-MPO- } \\
\text { ELISA }^{\text {b }}\end{array}$ & 6.4 & 1.7 & 30.8 & 69.2 & 98.3 & 75.0 & 97.7 \\
\hline
\end{tabular}

Abbreviations: ELISA, enzyme-linked immunosorbent assay; C-ANCA, cytoplasmic pattern of antineutrophil cytoplasmic antibodies; FN, false negative; FP, false positive; IIF, indirect immunofluorescence; MPO, myeloperoxidase; NPV, negative predictive value; P-ANCA, perinuclear pattern of antineutrophil cytoplasmic antibodies; pos, positive; PPV, positive predictive value; PR3, proteinase 3; SEN, sensitivity; SPF, specificity.

All data are expressed as \% (95\% confidence interval).

averall outcome of IIF as reference method.

${ }^{\mathrm{b} C} \mathrm{C}$-ANCA estimation by IIF as reference method.

CP-ANCA estimation by IIF as reference method.

without the categorical need for IIF.,9 Nonetheless, some researchers expressed the opinion that best results (specificity as high as $99 \%$ ) could be achieved by combining the results of IIF with that of ELISA (C-ANCA-PR3 ELISA and P-ANCA-MPO-ELISA). ${ }^{12-14}$ However, employing both the test may not be feasible in resource-limited diagnostic settings. In this study, we tried to assess the diagnostic accuracy of currently available ELISA technique compared with results of IIF.

We observed that both anti-PR3 and anti-MPO-ELISA had very high specificity ( - Table 3 ), taking corresponding IIF results as standard. Menezes et al found the sensitivity and specificity of ELISA to be 62 and 99\%, respectively, in concordance with our findings. ${ }^{12}$ However, Harris et al found ELISA to be technically superior to IIF with a specificity of $97 \%$ and a positive predictive value of $73 \%$, compared with $90 \%$ and $50 \%$ of IIF..$^{15}$ Similarly, another study also observed superior PPV of ELISA compared with that of ELISA ( 83 vs. $45 \%$ ). The likelihood ratio of a positive ELISA test was even higher than IIF (54.2 vs. 9.4) in this study. ${ }^{16}$ However, we found that ELISA suffered the drawback of low sensitivity and high false-negative results ( - Table 3). Our observations indicate that depending solely on ELISA for screening ANCA had the risk of missing substantial number of positive cases. Moreover, another study observed that detection of MPO-ANCA or PR3-ANCA without a positive IF rarely led to a new diagnosis of systemic vasculitis..$^{14}$

A good agreement was observed between the IIF and ELISA that indicates reliability on both methods. But a weaker agreement between C-ANCA and anti-PR3-ELISA compared with P-ANCA and anti-MPO-ELISA ( - Table 2 ) points toward more discordance in presence of C-ANCA and specific antigenicity toward PR3 antigen.

The study's limitation was that we could not correlate the two methods with histology findings as it is not routinely done in suspected AAV cases.

Thus, we conclude that currently used ELISA tests are highly specific, so false-positive results are least likely. Its sensitivity varies depending on the type of assay used but is generally inferior to IIFs. However, there is a good agreement between the results of IIF and ELISA. Suppose we use ELISA as the sole ANCA screening method in patients with symptoms suggestive of small vessel vasculitis, in that case, negative results have to be dealt with caution due to their high false negativity.

\section{Authors' Contribution}

S.D. is the lead and D.K. is the corresponding author. S.D. and D.K. drafted and conceptualized the manuscript; D.K. and G.S. were involved in the review and editing; S.D., R.S., U.S.R., and G.S. were involved in investigation; D.K., R.S., U.S.R., and S.D. were involved in methodology; S.D. and R.S. were involved in formal analysis; S.D., G.S., and D.K. were involved in project administration.

\section{Conflict of Interest}

None to declare.

\section{Acknowledgments}

We acknowledge All India Institute of Medical Sciences, Rishikesh, for allowing us to carry out the study. We are also thankful to the authority of All India Institute 
of Medical Sciences, Rishikesh, for providing with the resources to conduct this study.

\section{References}

1 Csernok E, Holle JU. Twenty-eight years with antineutrophil cytoplasmic antibodies (ANCA): how to test for ANCA - evidence-based immunology. ? Auto Immun Highlights 2010;1(1):39-43

2 Csernok E, Lamprecht P, Gross WL. Diagnostic significance of ANCAinvasculitis. NatClinPractRheumatol2006;2(4):174-175

3 Savige JA, Paspaliaris B, Silvestrini R, et al. A review of immunofluorescent patterns associated with antineutrophil cytoplasmic antibodies (ANCA) and their differentiation from other antibodies. J Clin Pathol 1998;51(8):568-575

4 Davies DJ, Moran JE, Niall JF, Ryan GB. Segmental necrotising glomerulonephritis with antineutrophil antibody: possible arbovirus aetiology? Br Med J (Clin Res Ed) 1982;285(6342) :60610.1136/bmj.285.6342.606

5 Bossuyt X, Cohen Tervaert JW, Arimura Y, et al. Position paper: Revised 2017 international consensus on testing of ANCAs in granulomatosis with polyangiitis and microscopic polyangiitis. Nat Rev Rheumatol 2017;13(11):683-692

6 Csernok E, Moosig F. Current and emerging techniques for ANCA detection in vasculitis. Nat Rev Rheumatol 2014;10(8):494-501

7 Sowa M, Grossmann K, Knütter I, et al. PLOS ONE Staff. Correction: simultaneous automated screening and confirmatory testing for vasculitis-specific ANCA. PLoS One 2015;10(3):e0120626

8 Allard-Chamard H, Liang P. Antineutrophil cytoplasmic antibodies testing and interpretation. Clin Lab Med 2019;39(4):539-552
9 Damoiseaux J, Csernok E, Rasmussen N, et al. Detection of antineutrophil cytoplasmic antibodies (ANCAs): a multicentre European Vasculitis Study Group (EUVAS) evaluation of the value of indirect immunofluorescence (IIF) versus antigen-specific immunoassays. Ann Rheum Dis 2017;76(4):647-653

10 Kirkwood BR, Sterne AC, Analysis of binary outcomes. In: Medical Statistics. 2nd edition. Malden, MA: Wiley Blackwell; 2009

11 Savige J, Gillis D, Benson E, et al. International consensus statement on testing and reporting of antineutrophil cytoplasmic antibodies (ANCA) Am J Clin Pathol 1999;111(4):507-513

12 Menezes JM, Rossener R, Silva APMD, Rodrigues SS, Mangueira CLP. Comparison between enzyme-linked immunosorbent assay and indirect immunofluorescence for detection of antineutrophil cytoplasmic antibodies. Einstein (Sao Paulo) 2020;18:eAO513210.31744/einstein_journal/2020A05132(São Paulo)

13 Schulte-Pelkum J, Radice A, Norman GL, et al. Novel clinical and diagnostic aspects of antineutrophil cytoplasmic antibodies. J Immunol Res 2014;2014:18541610.1155/2014/185416

14 Rao DA, Wei K, Merola JF, et al. Myeloperoxidase-antineutrophil cytoplasmic antibodies (MPO-ANCA) and proteinase 3-ANCA without immunofluorescent ANCA found by routine clinical testing. J Rheumatol 2015;42(5):847-852

15 Harris A, Chang G, Vadas M, Gillis D. ELISA is the superior method for detecting antineutrophil cytoplasmic antibodies in the diagnosis of systemic necrotising vasculitis. J Clin Pathol 1999;52(9):670-676

16 Stone JH, Talor M, Stebbing J, et al. Test characteristics of immunofluorescence and ELISA tests in 856 consecutive patients with possible ANCA-associated conditions. Arthritis Care Res 2000;13(6):424-434 